\title{
Поверхностно-излучающий квантово-каскадный лазер с кольцевым резонатором
}

\author{
(C) А.В. Бабичев ${ }^{1}$, Е.С. Колодезный ${ }^{1}$, А.Г. Гладышев ${ }^{1}$, Д.В. Денисов ${ }^{2}$, Г.В. Вознюк ${ }^{1,3}$, М.И. Митрофранов $^{3}$, \\ Н Ю. Харин ${ }^{4}$, В.Ю. Паневин ${ }^{4}$, С.О. Слипченко ${ }^{3}$, А.В. Лютецкий ${ }^{3}$, В.П. Евтихиев ${ }^{3}$, \\ Л.Я. Карачинский ${ }^{1,3,5}$, И.И. Новиков ${ }^{1,3,5}$, Н.А. Пихтин ${ }^{3}$, А.Ю. Егоров ${ }^{1}$ \\ ${ }^{1}$ Национальный исследовательский университет ИТМО, \\ 197101 Санкт-Петербург, Россия \\ ${ }^{2}$ Санкт-Петербургский государственный электротехнический университет „ЛЭТИ“ им. В.И. Ульянова (Ленина), \\ 197022 Санкт-Петербург, Россия \\ ${ }^{3}$ Физико-технический институт им. А.Ф. Иоффре Российской академии наук, \\ 194021 Санкт-Петербург, Россия \\ ${ }^{4}$ Санкт-Петербургский политехнический университет Петра Великого, \\ 195251 Санкт-Петербург, Россия \\ ${ }^{5} \mathrm{OOO} \mathrm{„Коннектор} \mathrm{Оптикс“,}$ \\ 194292 Санкт-Петербург, Россия \\ E-mail: a.babichev@mail.ioffe.ru
}

Поступила в Редакцию 4 марта 2021 г.

В окончательной редакции 12 марта 2021 г.

Принята к публикации 12 марта 2021 г.

\begin{abstract}
Представлены результаты исследований квантово-каскадных лазеров с поверхностным выводом излучения через дифракционную решетку, сформированную в слоях верхней обкладки волновода методом ионнолучевого травления. Активная область гетероструктуры лазера была сформирована на основе гетеропары твердых растворов $\operatorname{In}_{0.53} \mathrm{Ga}_{0.47} \mathrm{As} / \mathrm{Al}_{0.48} \mathrm{In}_{0.52} \mathrm{As}$ с двухфононным опустошением нижнего уровня в каскаде. Для лазера с диаметром кольцевого резонатора 191 мкм продемонстрирована лазерная генерация при комнатной температуре вблизи 7.9 мкм. Величина межмодового расстояния в спектрах генерации данного типа лазеров соответствует модам шепчущей галереи.
\end{abstract}

Ключевые слова: сверхрешетки, квантово-каскадный лазер, эпитаксия, фосфид индия, кольцевой резонатор.

DOI: 10.21883/FTP.2021.07.51026.9644

\section{1. Введение}

В силу поперечно-магнитной (ТМ) поляризации излучения в лазерах на межподзонных переходах, реализация поверхностного вывода излучения в квантово-каскадных лазерах (ККЛ) в конструкции микрорезонатора с распределенными брэгговскими резонаторами (в конструкции вертикально-излучающего лазера) невозможна [1-3]. В свою очередь формирование дифракционной решетки 2-го порядка позволяет реализовать поверхностно-излучающий ККЛ [4].

К настоящему моменту успешно реализованы поверхностно-излучающие ККЛ среднего инфракрасного диапазона $[5,6]$, в том числе, в конструкции с кольцевым резонатором [7-13]. Интерес к исследованиям ККЛ с кольцевым резонатором обусловлен в первую очередь возможностью их применения при детектировании и анализе химических газов [14]. Более того, малые геометрические размеры позволяют их интегрировать с миниатюрными полыми волноводами (substrate-integrated hollow waveguides), что позволяет создавать системы одновременного детектирования нескольких газов в смеси [15]. Кроме того, в последнее время наблюдается значительный интерес в области исследования частотных гребенок на основе ККЛ с кольцевым резонатором $[12,13]$.
Традиционно, при формировании селективного кольцевого резонатора используется метод сухого ионного травления слоев верхней обкладки волновода [4] либо селективное жидкостное травление слоя InGaAs до стопслоя на основе InP, расположенного на поверхности активной области с последующим шагом заращивания слоями верхней обкладки волновода [7].

В настоящей работе представлены результаты по формированию и изучению характеристик поверхностноизлучающих ККЛ спектрального диапазона 7.5-8.0 мкм с кольцевым резонатором на основе дифракционной решетки 2-го порядка, сформированной методом ионнолучевого травления.

\section{2. Эксперимент}

Гетероструктура ККЛ была выращена на подложке InP c ориентацией (001) компанией „Коннектор Оптикс“ на промышленной установке молекулярно-пучковой эпитаксии Riber 49 [16,17]. Использована конструкция волновода с верхней обкладкой толщиной 3.9 мкм на основе фосфида индия (концентрация $n=1.0 \cdot 10^{17} \mathrm{~cm}^{-3}$ ). Активная область сформирована на основе гетеропары твердых растворов $\operatorname{In}_{0.53} \mathrm{Ga}_{0.47} \mathrm{As} / \mathrm{Al}_{0.48} \mathrm{In}_{0.52} \mathrm{As}$ c двухфононным опустошением нижнего уровня в каска- 

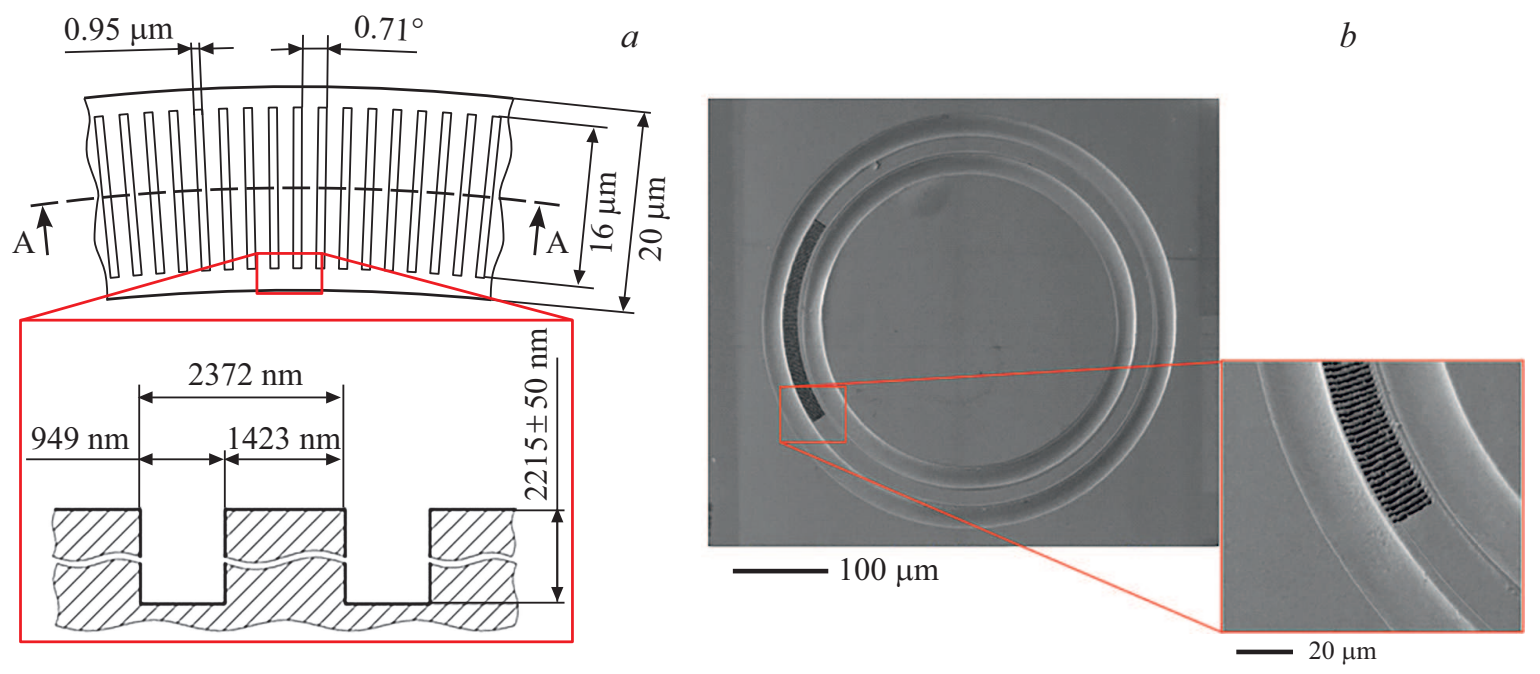

Рис. 1. $a-$ схематический вид сегмента решетки, сформированной в слоях верхней обкладки волновода ККЛ (вид сверху и сечение); $b$ - СЭМ-изображение ККЛ в конструкции с кольцевым резонатором (вид сверху), а также увеличенное СЭМизображение области с вытравленной дифракционной решеткой (вид сверху).

де $[18,19]$. В качестве контактных слоев использованы слои $\operatorname{In}_{0.53} \mathrm{Ga}_{0.47}$ As толщиной 100 и 20 нм с уровнями легирования $1.0 \cdot 10^{17}$ и $1.0 \cdot 10^{19} \mathrm{~cm}^{-3}$ соответственно. Формирование кристалла ККЛ проводилось по методике, аналогичной описанной ранее [20]. Использована конструкция глубокой мезы с затравом в подложку InP. Внешний радиус кольцевого резонатора составил 201 мкм. Ширина резонатора вблизи поверхности составила 20 мкм. Монтаж лазерного кристалла проводился подложкой на медный теплоотвод при помощи индиевого припоя.

Травление дифракционной решетки, обеспечивающей распределенную обратную связь (РОС), проводилось в сверхвысоком вакууме фокусированным пучком ионов галлия с энергией 30 кэВ и рабочем токе 490 пА, сфокусированным в пятно диаметром 40 нм [21]. Доза облучения области дифракционной решетки при травлении составляла $1.4 \cdot 10^{11} \mathrm{\Pi K} / \mathrm{cm}^{2}$. Период дифракционной решетки 2-го порядка составил 2.37 мкм (в угловых координатах $0.71^{\circ}$ ). Скважность штрихов решетки составила $\sim 60 \%$. Глубина травления штрихов составила $2215 \pm 50$ нм (см. pис. $1, a$ ) и приведена с учетом толщины верхней металлизации на основе слоев Ti-Pt-Au c суммарной толщиной $\sim 605$ нм. Изображения сканирующей электронной микроскопии (СЭМ) ККЛ в конструкции с кольцевым резонатором представлены на рис. $1, b$.

Детектирование оптического сигнала производилось с помощью фотодиода $\mathrm{HgCdTe}$, охлаждаемого жидким азотом. Быстродействие фотодетектора составляло $\sim 100$ нс. Спектры стимулированного излучения измерялись в режиме пошагового сканирования с помощью фурье-спектрометра Bruker Vertex 80v. Спектральное разрешение составляло $0.2 \mathrm{~cm}^{-1}$. При исследовании спектров излучения длительность импульсов тока накач-
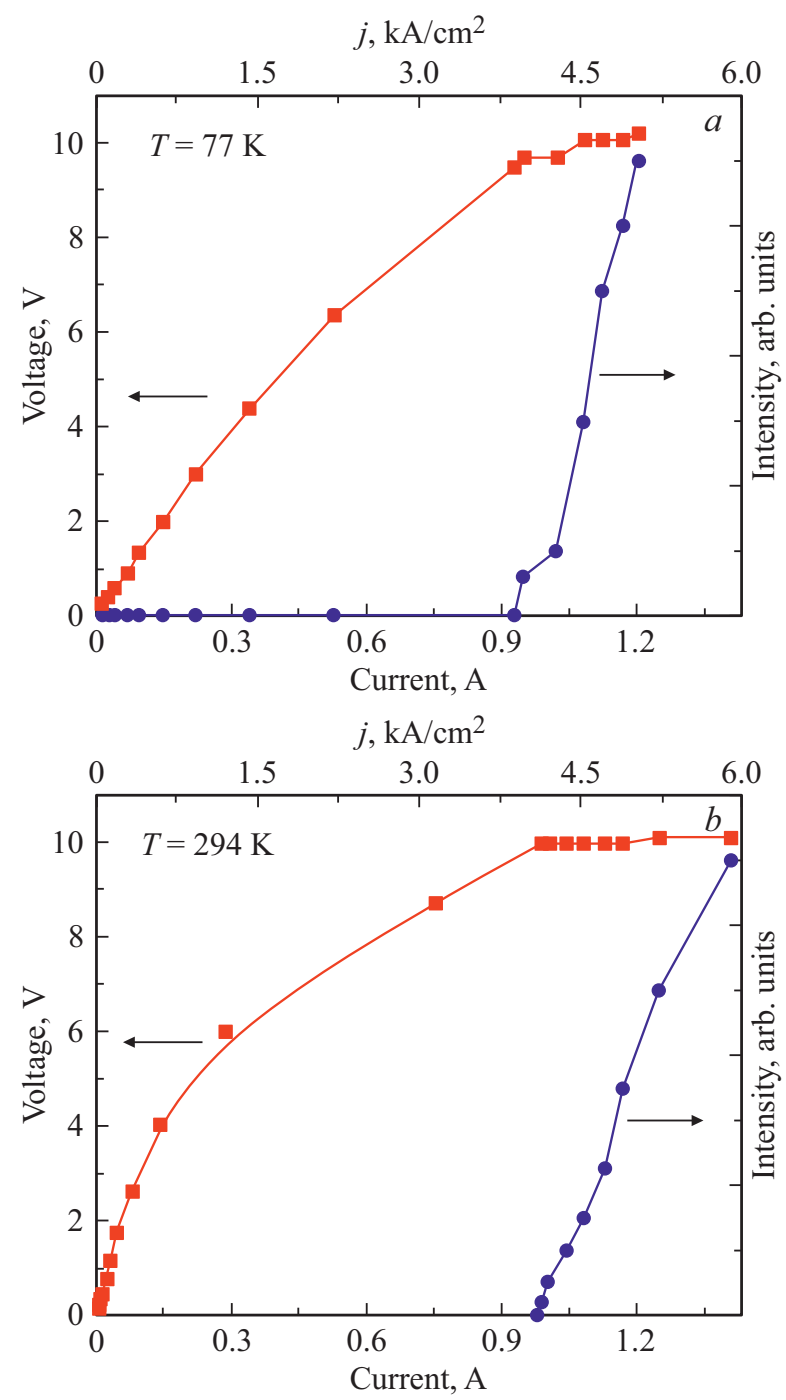

Рис. 2. Вольт-амперные характеристики исследуемого ККЛ (левая ось ординат), а также зависимость интегральной интенсивности от тока накачки (правая ось ординат), измеренные при $77 \mathrm{~K}$ (панель $a$ ) и $294 \mathrm{~K}$ (панель $b$ ). 

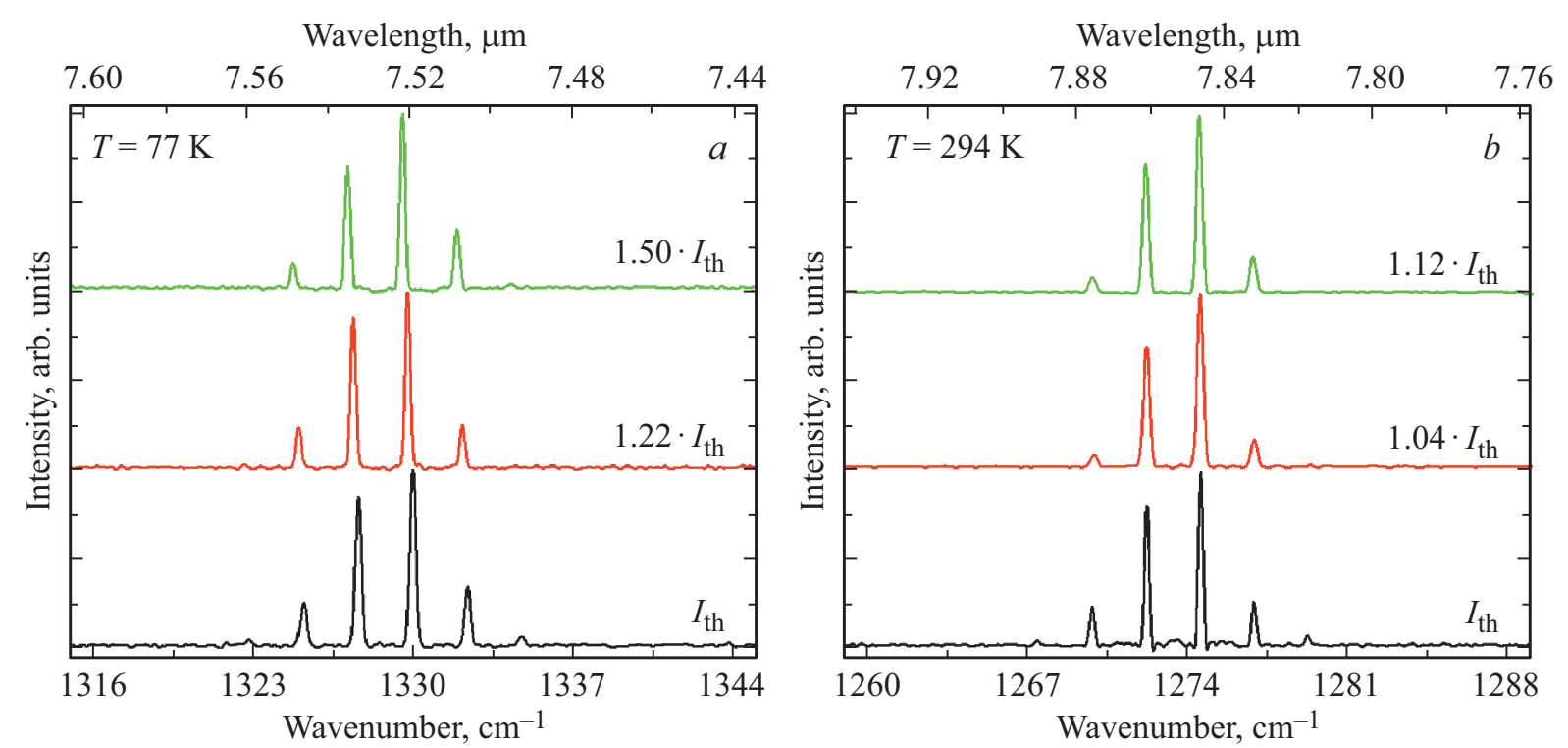

Рис. 3. $a$ - отнормированные спектры поверхностной генерации исследуемого ККЛ, измеренные при температуре $77 \mathrm{~K}$ и различных уровнях токовой накачки; $b$ - отнормированные спектры поверхностной генерации исследуемого ККЛ, измеренные при температуре $294 \mathrm{~K}$ и различных уровнях токовой накачки.

ки $\tau$ составляла 200 нс, а при исследовании вольт-, ваттамперных характеристик $\tau=70$ нс. Частота следования импульсов $f=15$ кГц. Измерения проведены при температурах 77 и $294 \mathrm{~K}$.

\section{3. Результаты и их обсуждение}

На рис. 2 представлены вольт- и ватт-амперные характеристики, измеренные при температурах 77 и $294 \mathrm{~K}$. Пороговый ток $I_{\mathrm{th}}$, измеренный при температуре $77 \mathrm{~K}$, составил $0.9 \mathrm{~A}$, что соответствует пороговой плотности тока $j_{\text {th }}=3.9$ кА/см ${ }^{2}$. Увеличение температуры до $294 \mathrm{~K}$ приводит к весьма слабому увеличению $I_{\text {th }}$ до $1.0 \mathrm{~A}$ (соответствует $j_{\text {th }}=4.0 \kappa \mathrm{A} / \mathrm{cm}^{2}$ ). Слабое изменение порогового тока с температурой, по-видимому, связано с наличием температурно-независимых оптических потерь, определяемых геометрией резонатора, которые и определяют величину порогового тока. При этом усиление в ККЛ достаточно для получения генерации в широком температурном диапазоне. Для оценки оптических потерь, вносимых при вытравливании дифракционной решетки и выводе излучения через решетку, проведено измерение вольт-, ватт-амперных характеристик и определение плотности порогового тока лазера в конструкции резонатора Фабри-Перо с длиной 1.5 мм, близкой к длине ККЛ с кольцевым резонатором (1.2 мм). Величина $j_{\text {th }}$ полоскового лазера составила 3.7 кА/ $\mathrm{cm}^{2}$. Сравнимые значения плотностей порогового тока лазера ККЛ с кольцевым резонатором и полоскового лазера позволяют сделать вывод, что оптические потери, вносимые при вытравливании дифракционной решетки, сопоставимы с оптическими потерями на зеркалах в полосковом лазере, $\sim 9 \mathrm{~cm}^{-1}$ [17]. Произведена оценка полных оптических потерь в кольцевом ККЛ, которые составили $13 \mathrm{~cm}^{-1}$. Оценена добротность резонатора $Q=2006$

На рис. 3 представлены спектры лазерной генерации поверхностно-излучающего ККЛ с кольцевым резонатором. При температуре $77 \mathrm{~K}$ лазерная генерация наблюдается вблизи 7.5 мкм (см. рис. 3,a). При пороговом значении тока накачки в спектре генерации присутствует 6 оптических мод. Повышение уровня накачки до $1.5 \cdot I_{\text {th }}$ приводит к уменьшению числа оптических мод до 4. Межмодовое расстояние $\Delta \lambda$ соответствует случаю мод шепчущей галереи, для которых $\Delta \lambda=\lambda^{2} /\left(2 \pi R_{\text {out }}\right) n_{\mathrm{gr}}$, где $n_{\mathrm{gr}}-$ групповой показатель преломления, $R_{\text {out }}-$ внешний радиус кольцевого резонатора, $\lambda$ - длина волны излучения [7,22]. С увеличением температуры до $294 \mathrm{~K}$ наблюдается длинноволновый сдвиг, что может быть обусловлено уменьшением разрыва зоны проводимости на гетерогранице с ростом температуры [23,24]. При температуре $294 \mathrm{~K}$ лазерная генерация наблюдается вблизи длины волны 7.85 мкм (см. рис. 3, $b$ ). При пороговом значении тока накачки в спектре генерации присутствует 6 оптических мод. Повышение уровня накачки приводит к уменьшению числа мод до 4.

\section{4. Заключение}

В ходе исследований представлены результаты по формированию и изучению характеристик поверхностно-излучающих ККЛ спектрального диапазона 7.5-8.0 мкм с кольцевым резонатором на основе дифракционной решетки 2-го порядка, сформированной методом ионно-лучевого травления. Продемонстрирована поверхностная лазерная генерация ККЛ с кольце- 
вым резонатором при комнатной температуре. Лазерная генерация наблюдалась вблизи длины волны 7.9 мкм. Продемонстрирована низкая плотность порогового тока $j_{\text {th }}=4.0 \kappa \mathrm{A} / \mathrm{cm}^{2}$ в сравнении с результатами численных оценок [22]. Величина межмодового расстояния соответствует случаю мод шепчущей галереи. Дальнейшие исследования будут направлены на увеличение величины оптического связывания с целью реализации поверхностно-излучающего ККЛ, работающего в одночастотном режиме.

\section{Финансирование работы}

Работа выполнена за счет гранта Российского научного фонда (проект № 20-79-10285).

\section{Конфликт интересов}

Авторы заявляют, что у них нет конфликта интересов.

\section{Список литературы}

[1] A.V. Babichev, L.Y. Karachinsky, I.I. Novikov, A.G. Gladyshev, S. Mikhailov, V. Iakovlev, A. Sirbu, G. Stepniak, L. Chorchos, J.P. Turkiewicz, M. Agustin, N.N. Ledentsov, K.O. Voropaev, A.S. Ionov, A.Y. Egorov. Proc. SPIE, 10122, 1012208 (2017). https://doi.org/10.1117/12.2250842

[2] A.V. Babichev, L.Ya. Karachinsky, I.I. Novikov, A.G. Gladyshev, S.A. Blokhin, S. Mikhailov, V. Iakovlev, A. Sirbu, G. Stepniak, L. Chorchos, J.P. Turkiewicz, K.O. Voropaev, A.S. Ionov, M. Agustin, N.N. Ledentsov, A.Yu. Egorov. IEEE J. Quantum Electron., 53 (6), 1 (2017).

[3] L. Chorchos, N.N. Ledentsov, J.R. Kropp, V.A. Shchukin, V.P. Kalosha, A. Lewandowski, J.P. Turkiewicz, N.N. Ledentsov. J. Lightwave Technol., 38 (7), 1747 (2020).

[4] D.H. Wu, M. Razeghi. APL Mater., 5 (3), 035505 (2017).

[5] Y. Liang, Z. Wang, J. Wolf, E. Gini, M. Beck, B. Meng, J. Faist, G. Scalari. Appl. Phys. Lett., 114 (3), 031102 (2019).

[6] Z. Wang, Y. Liang, B. Meng, Y.-T. Sun, G. Omanakuttan, E. Gini, M. Beck, I. Sergachev, S. Lourdudoss, J. Faist, G. Scalari. Opt. Express, 27 (16), 22708 (2019).

[7] Y. Bai, S. Tsao, N. Bandyopadhyay, S. Slivken, Q.Y. Lu, D. Caffey, M. Pushkarsky, T. Day, M. Razeghi. Appl. Phys. Lett., 99 (26), 261104 (2011).

[8] R. Szedlak, M. Holzbauer, D. MacFarland, T. Zederbauer, H. Detz, A.M. Andrews, C. Schwarzer, W. Schrenk, G. Strasser. Sci. Rep., 5 (1), 16668 (2015).

[9] R. Szedlak, T. Hisch, B. Schwarz, M. Holzbauer, D. MacFarland, T. Zederbauer, H. Detz, A.M. Andrews, W. Schrenk, S. Rotter, G. Strasser. Sci. Rep., 8 (1), 1 (2018).

[10] B. Hinkov, J. Hayden, R. Szedlak, P. Martin-Mateos, B. Jerez, P. Acedo, G. Strasser, B. Lendl. Opt. Express, 27 (10), 14716 (2019).

[11] H. Knötig, B. Hinkov, R. Weih, S. Höfling, J. Koeth, G. Strasser. Appl. Phys. Lett., 116 (13), 131101 (2020).

[12] M. Piccardo, B. Schwarz, D. Kazakov, M. Beiser, N. Opačak, Y. Wang, S. Jha, J. Hillbrand, M. Tamagnone, W.T. Chen, A.Y. Zhu, L.L. Columbo, A. Belyanin, F. Capasso. Nature, 582, 360 (2020).
[13] B. Meng, M. Singleton, M. Shahmohammadi, F. Kapsalidis, R. Wang, M. Beck, J. Faist. Optica, 7 (2), 162 (2020).

[14] R. Szedlak, J. Hayden, P. Martín-Mateos, M. Holzbauer, A. Harrer, B. Schwarz, B. Hinkov, D. MacFarland, T. Zederbauer, H. Detz, A.M. Andrews, W. Schrenk, P. Acedo, B. Lendl, G. Strasser. Opt. Eng., 57 (1), 1 (2017).

[15] E. Tütüncü, V. Kokoric, R. Szedlak, D. MacFarland, T. Zederbauer, H. Detz, A.M. Andrews, W. Schrenk, G. Strasser, B. Mizaikoff. Analyst, 141 (22), 6202 (2016).

[16] A.V. Babichev, A.G. Gladyshev, A.S. Kurochkin, E.S. Kolodeznyi, G.S. Sokolovskii, V.E. Bougrov, L.Ya. Karachinsky, I.I. Novikov, A.G. Bousseksou, A.Yu. Egorov. Semiconductors, 52 (8), 1082 (2018).

[17] A.V. Babichev, V.V. Dudelev, A.G. Gladyshev, D.A. Mikhailov, A.S. Kurochkin, E.S. Kolodeznyi, V.E. Bougrov, V.N. Nevedomskiy, L.Ya. Karachinsky, I.I. Novikov, D.V. Denisov, A.S. Ionov, S.O. Slipchenko, A.V. Lutetskiy, N.A. Pikhtin, G.S. Sokolovskii, A.Yu. Egorov. Semiconductors, 45 (7), 735 (2019).

[18] A.V. Babichev, A.S. Kurochkin, E.C. Kolodeznyi, A.V. Filimonov, A.A. Usikova, V.N. Nevedomsky, A.G. Gladyshev, L.Ya. Karachinsky, I.I. Novikov, A.Yu. Egorov. Semiconductors, 52 (6), 745 (2018).

[19] A.V. Babichev, A.G. Gladyshev, A.S. Kurochkin, V.V. Dudelev, E.S. Kolodeznyi, G.S. Sokolovskii, V.E. Bugrov, L.Y. Karachinsky, I.I. Novikov, D.V. Denisov, A.S. Ionov, S.O. Slipchenko, A.V. Lyutetskii, N.A. Pikhtin, A.Y. Egorov. Tech. Phys. Lett., 45 (4), 398 (2019).

[20] V.V. Dudelev, D.A. Mikhailov, A.V. Babichev, A.D. Andreev, S.N. Losev, E.A. Kognovitskaya, Yu.K. Bobretsova, S.O. Slipchenko, N.A. Pikhtin, A.G. Gladyshev, D.V. Denisov, I.I. Novikov, L.Ya. Karachinsky, V.I. Kuchinskii, A.Yu. Egorov, G.S. Sokolovskii. Quantum Electron., 50 (2), 141 (2020).

[21] A.V. Babichev, D.A. Pashnev, A.G. Gladyshev, D.V. Denisov, G.V. Voznyuk, L.Y. Karachinsky, I.I. Novikov, M.I. Mitrofanov, V.P. Evtikhiev, D.A. Firsov, L.E. Vorob'ev, N.A. Pikhtin, A.Y. Egorov. Tech. Phys. Lett., 46 (4), 312 (2020).

[22] A.V. Babichev, D.A. Pashnev, A.G. Gladyshev, A.S. Kurochkin, E.S. Kolodeznyi, L.Ya. Karachinsky, I.I. Novikov, D.V. Denisov, V.V. Dudelev, G.S. Sokolovskii, D.A. Firsov, L.E. Vorob’ev, S.O. Slipchenko, A.V. Lutetskiy, N.A. Pikhtin, A.Yu. Egorov. Opt. Spectrosc., 128 (8), 1187 (2020).

[23] Y. Bai, S. Slivken, S. Kuboya, S.R. Darvish, M. Razeghi. Nat. Photonics, 4 (2), 99 (2010).

[24] A.V. Babichev, A.G. Gladyshev, D.V. Denisov, L.Y. Karachinsky, I.I. Novikov, L. Boulley, A. Bousseksou, N.A. Pikhtin, A.Y. Egorov. Opt. Spectrosc., 127 (2), 279 (2019).

Редактор Г.А. Оганесян 


\section{Surface emitting range quantum-cascade ring laser}

A.V. Babichev ${ }^{1}$, E.S. Kolodeznyi ${ }^{1}$, A.G. Gladyshev' ${ }^{1}$, D.V. Denisov' ${ }^{2}$, G.V. Voznyuk ${ }^{1,3}$, M.I. Mitrofanov ${ }^{3}$, N.Yu. Kharin ${ }^{4}$, V.Yu. Panevin ${ }^{4}$, S.O. Slipchenko ${ }^{3}$, A.V. Lyutetskii ${ }^{3}$, V.P. Evtikhiev ${ }^{3}$, L.Ya. Karachinsky 1,3,5, I.I. Novikov ${ }^{1,3,5}$, N.A. Pikhtin ${ }^{3}$, A.Yu. Egorov ${ }^{1}$

${ }^{1}$ ITMO University,

197101 St. Petersburg, Russia

${ }^{2}$ St. Petersburg Electrotechnical University „LETl“, 197022 St. Petersburg, Russia

${ }^{3}$ loffe Institute,

194021 St. Petersburg, Russia

${ }^{4}$ Peter the Great St. Petersburg Polytechnic University, 195251 St. Petersburg, Russia

5 "Connector Optics" LLC,

194292 St. Petersburg, Russia

Abstract The results of studies of quantum-cascade laser with a surface emission through a grating formed in the layers of the top cladding of the waveguide by ion beam milling are presented. The active region of the QCL heterostructure was formed based on a heteropair of $\operatorname{In}_{0.53} \mathrm{Ga}_{0.47} \mathrm{As} / \mathrm{Al}_{0.48} \mathrm{In}_{0.52} \mathrm{As}$ solid alloys with two-phonon resonance design. It is shown that lasing at room temperature close to $7.9 \mu \mathrm{m}$ is demonstrated for a laser with a ring diameter of $191 \mu \mathrm{m}$. The mode spacing corresponds to whispering gallery modes. 